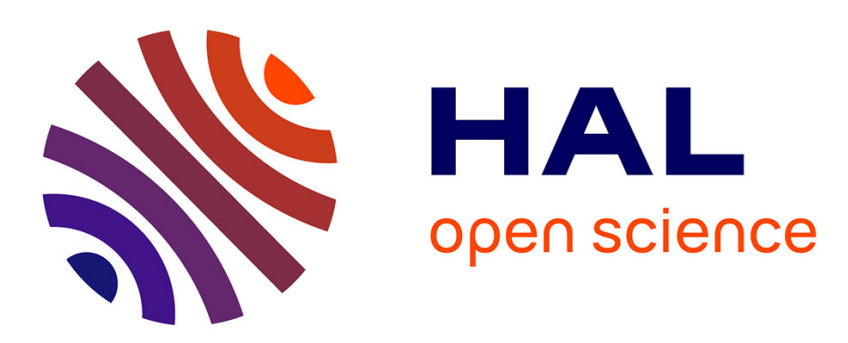

\title{
Analysis of electrodes' placement and deformation in deep brain stimulation from medical images
}

Maroua Mehri, Florent Lalys, Camille Maumet, Claire Haegelen, Pierre

Jannin

\section{- To cite this version:}

Maroua Mehri, Florent Lalys, Camille Maumet, Claire Haegelen, Pierre Jannin. Analysis of electrodes' placement and deformation in deep brain stimulation from medical images. SPIE Medical Imaging, Feb 2012, San diego, CA, United States. pp.8316-32, 10.1117/12.912225 . inserm-00669674

\section{HAL Id: inserm-00669674 https://www.hal.inserm.fr/inserm-00669674}

Submitted on 2 Dec 2015

HAL is a multi-disciplinary open access archive for the deposit and dissemination of scientific research documents, whether they are published or not. The documents may come from teaching and research institutions in France or abroad, or from public or private research centers.
L'archive ouverte pluridisciplinaire HAL, est destinée au dépôt et à la diffusion de documents scientifiques de niveau recherche, publiés ou non, émanant des établissements d'enseignement et de recherche français ou étrangers, des laboratoires publics ou privés. 


\title{
Analysis of electrodes' placement and deformation in deep brain stimulation from medical images
}

\author{
Maroua Mehri ${ }^{1,2}$, Florent Lalys ${ }^{1,2}$, Camille Maumet ${ }^{1,2}$, Claire Haegelen ${ }^{1,2,3}$, Pierre Jannin ${ }^{1,2}$ \\ 1 \\ INSERM, U746, Faculty of Medicine CS 34317, F-35043 Rennes, France \\ ${ }^{2}$ University of Rennes I, CNRS, UMR 6074, IRISA, F-35042 Rennes, France \\ ${ }^{3}$ Department of Neurosurgery, Pontchaillou University Hospital, F-35043 Rennes, France
}

\begin{abstract}
Deep brain stimulation (DBS) is used to reduce the motor symptoms such as rigidity or bradykinesia, in patients with Parkinson's disease (PD). The Subthalamic Nucleus (STN) has emerged as prime target of DBS in idiopathic PD. However, DBS surgery is a difficult procedure requiring the exact positioning of electrodes in the pre-operative selected targets. This positioning is usually planned using patients' pre-operative images, along with digital atlases, assuming that electrode's trajectory is linear. However, it has been demonstrated that anatomical brain deformations induce electrode's deformations resulting in errors in the intra-operative targeting stage. In order to meet the need of a higher degree of placement accuracy and to help constructing a computer-aided-placement tool, we studied the electrodes' deformation in regards to patients' clinical data (i.e., sex, mean PD duration and brain atrophy index). Firstly, we presented an automatic algorithm for the segmentation of electrode's axis from post-operative CT images, which aims to localize the electrodes' stimulated contacts. To assess our method, we applied our algorithm on 25 patients who had undergone bilateral STNDBS. We found a placement error of $0.91 \pm 0.38 \mathrm{~mm}$. Then, from the segmented axis, we quantitatively analyzed the electrodes' curvature and correlated it with patients' clinical data. We found a positive significant correlation between mean curvature index of the electrode and brain atrophy index for male patients and between mean curvature index of the electrode and mean PD duration for female patients. These results help understanding DBS electrode' deformations and would help ensuring better anticipation of electrodes' placement.
\end{abstract}

\section{KEYWORDS}

Deep Brain Stimulation, neurosurgery, Parkinson's disease, targeting accuracy, segmentation, mean curvature and brain atrophy.

\section{1 - INTRODUCTION}

PD is a degenerative neurological disease that results from the slow and progressive death of neurons in the brain ${ }^{[1]}$. DBS is currently the most favored treatment of Parkinson's patients and specifically for patients with advanced disease whose symptoms do not respond in a stable manner to L-Dopa ${ }^{[2,3]}$. DBS at STN showed very good results on Parkinson's motor symptoms ${ }^{[4]}$. Although it has shown its effectiveness, it also presents limitations: it can cause several types of neuropsychological disorders ${ }^{[5,6,7,8,9]}$. Indeed, the accuracy of placement is crucial to avoid unwanted stimulation of non-target portions being located in the STN's vicinity. In addition, the exact positioning of the electrode is usually planned assuming that the electrode's trajectory is linear. Nevertheless, recent works ${ }^{[10,11,12]}$ showed that brain shift causes deformations of electrodes and consequent targeting errors. To better define electrode's deformations, we used an automatic electrode and contacts segmentation algorithm described below. To better understand electrode's deformations, we correlated electrode's curvature with patients' clinical data.

\section{2 - MATERIALS AND METHODS}

The studied population consisted of 25 patients (12 female and 13 male, mean age $56 \pm 8$ years, mean duration's PD $12 \pm 5$ years) with idiopathic PD who had undergone bilateral STN-DBS. The implanted electrode is the quadripolar model 3389 (Medtronic Sofamor Danek), with four contacts representing platinum-iridium cylindrical surfaces $(1.27 \mathrm{~mm}$ diameter, $1.5 \mathrm{~mm}$ long, and spaced each of the other of $0.5 \mathrm{~mm}$ ). For this study, we used post-operative CT scans images $(0.44 \mathrm{~mm} \times 0.44 \mathrm{~mm} \times 0.6 \mathrm{~mm}$ in post-operative acquisitions, GE Healthcare VCT 64) and pre-operative 3-T 
T1-weighted MR (1 mm x $1 \mathrm{~mm}$ x $1 \mathrm{~mm}$, Philips Medical Systems). Preprocessing consisted in denoising pre-operative MRI and post-operative $\mathrm{CT}$ with the non-local means algorithm ${ }^{[13]}$.

\subsection{Automatic contact localization algorithm}

An automatic contact localization algorithm, based on the segmentation of electrode's axis from post-operative images, was developed to determine the spatial coordinates of the contacts for each electrode. Firstly, the post-operative CT scan was linearly registered to a reference CT with an affine transformation (algorithm: Newuoa, cost function: normalized mutual information, interpolation: Spline3) ${ }^{[14,15]}$, where a Region Of interest (ROI) including deep brain structures was preliminary defined. After the registration, we extracted coordinates of hypo-signal artefacts (i.e., white artefacts) using a threshold for each slice of the ROI. This segmentation task then permitted us to determine the centers of each hypo-signal region for each slice after adding a filter to retrieve the connected components. We obtained a points cloud modeling the electrode's axis, illustrated in Figure 1 (green points). From it, we computed the characteristic polynomial (second degree which has the following form: $z=a x^{2}+b y^{2}+c x y+d x+e y+f$, where $a, b, c, d, e$, and $f$ are constants and $\mathrm{x}, \mathrm{y}$, and $\mathrm{z}$ represent respectively the value of the $\mathrm{x}$-axis, $\mathrm{y}$-axis, and $\mathrm{z}$-axis) of electrode's curve with multiple linear regression determined along the length of electrode. Finally, we computed coordinates of the contact center, illustrated in Figure 1 (blue points), from the characteristic polynomial of electrode's curve, in accordance with the original electrode settings (model: 3389), such as the contact's length $(1.5 \mathrm{~mm})$ or the distance between 2 contacts $(1.5 \mathrm{~mm})$, as the $\mathrm{x}$-axis (lateral) is oriented from right to left, $\mathrm{y}$-axis (antero-posterior) is oriented in the front (anterior) to the rear (posterior), and z-axis (vertical) is directed upwards.

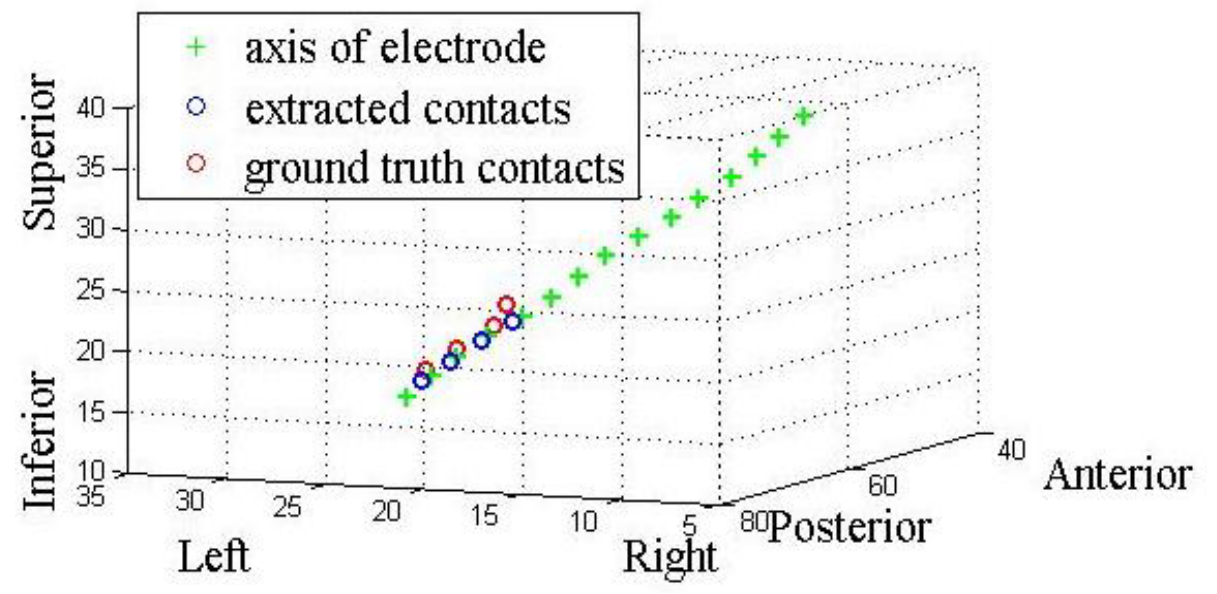

(a)

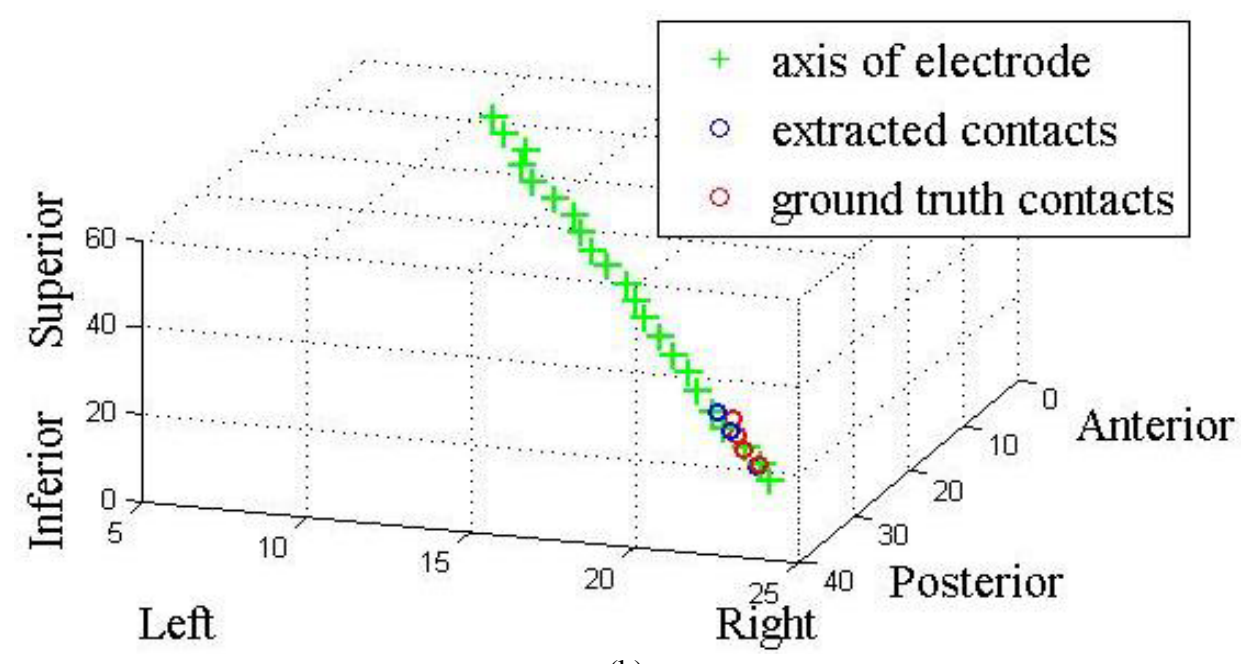

(b)

Figure 1: Electrode's axis, the 4 extracted contacts and the 4 ground truth contacts for left (a) and right (b) brain hemispheres of one patient 


\subsection{Analysis of electrodes' deformation}

With the method previously mentioned, the electrode's mean curvature was computed according to the formula ${ }^{[16]}$ representing the polynomial characteristic of electrode's curve.

Then, the mean curvature index $(\mathrm{MCI})$ is given by:

$$
\mathrm{MCI}=\frac{\mathrm{mP}-2 \mathrm{nN}+\mathrm{pM}}{2\left(\mathrm{MP}-\mathrm{N}^{2}\right)}
$$

where $\mathrm{M}, \mathrm{N}$ and $\mathrm{P}$ are coefficients of the first fundamental form and $\mathrm{m}, \mathrm{n}$ and $\mathrm{p}$ are coefficients of the second fundamental form.

Finally, we studied the correlation between the MCI and patients' clinical data: sex, age, PD duration and brain atrophy index. This index was estimated from pre-operative MR images by using SPM8 (Statistical Parametric Mapping; Wellcome Department of Imaging Neuroscience, University College London; www.fil.ion.ucl.ac.uk). The pre-operative MR images were segmented in grey matter (GM), white matter (WM), and cerebrospinal fluid (CSF) using the unified segmentation framework available in SPM8 ${ }^{[17]}$ (see Figure 2).

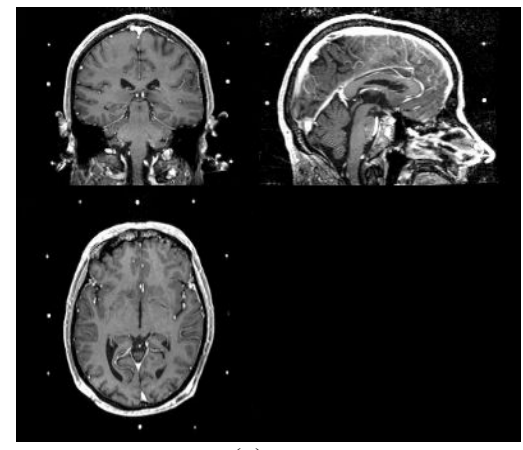

(a)

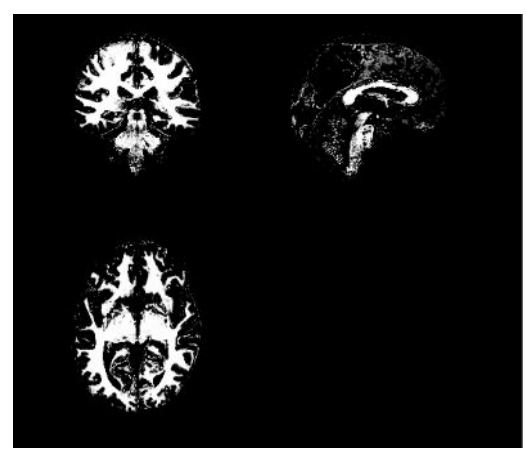

(c)

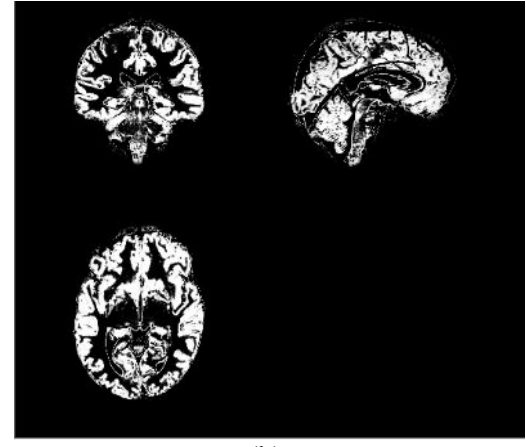

(b)

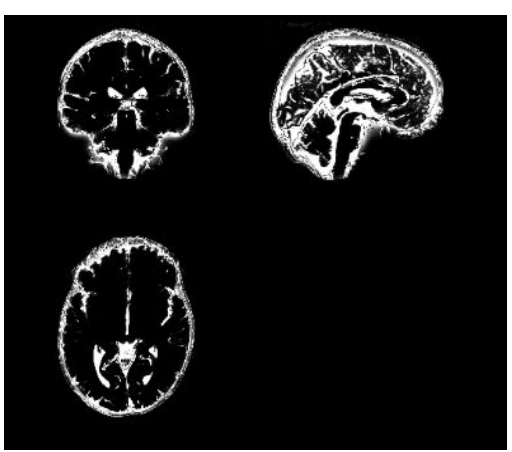

(d)

Figure 2: Segmentation of pre-operative MR image (a) in grey matter (b), white matter (c), and cerebrospinal fluid (d)

We computed a relative brain atrophy index (AI) by:

$$
\mathrm{AI}=\frac{\mathrm{GM}+\mathrm{WM}+\mathrm{CSF}}{\mathrm{GM}+\mathrm{WM}}
$$

where GM, WM, and CSF are volumes in $\mathrm{mL}$.

Correlation between the MCI and patients' clinical data was determined by computing cross-correlation' coefficients. In order to check the linearity between two variables and identify the statistical significance, we used Fstatistics for the regression and subsequently determined p-value. Level of significance was set at $p$-value $<0.05$. 


\section{3 - RESULTS}

\subsection{Automatic contact localization algorithm}

The developed contact localization algorithm was applied on each post-operative CT to compute contacts coordinates. We considered, as the reference, the coordinates taken on the post-operative CT image localized from hyper-signal artefacts (black artefacts) ${ }^{[18]}$. Euclidean distance between coordinates determined by our method and the reference was computed for the 25 patients, in order to estimate the global misplacement error. We found a placement error of $0.91 \pm 0.38 \mathrm{~mm}$. Three patients had bilateral contact coordinates which seemed to be aberrant due to an inaccurate local registration step. Figure 3 shows contacts' centers in the post-operative CT image of one patient, determined automatically by our algorithm.

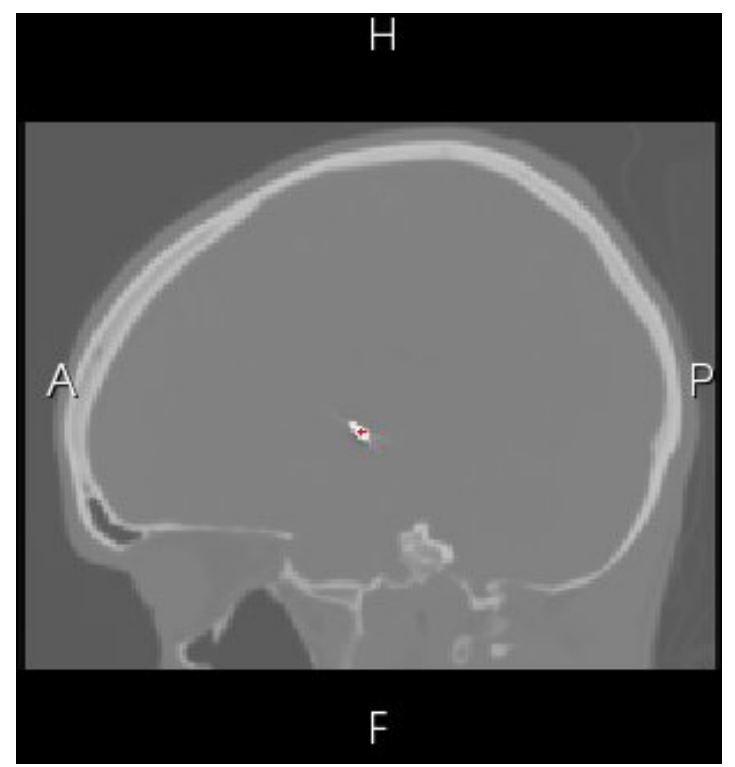

(a)

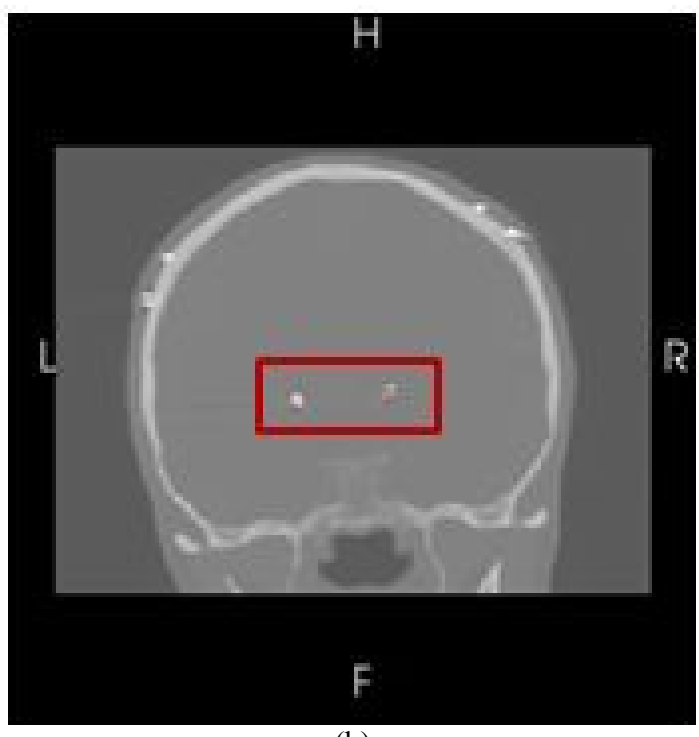

(b)

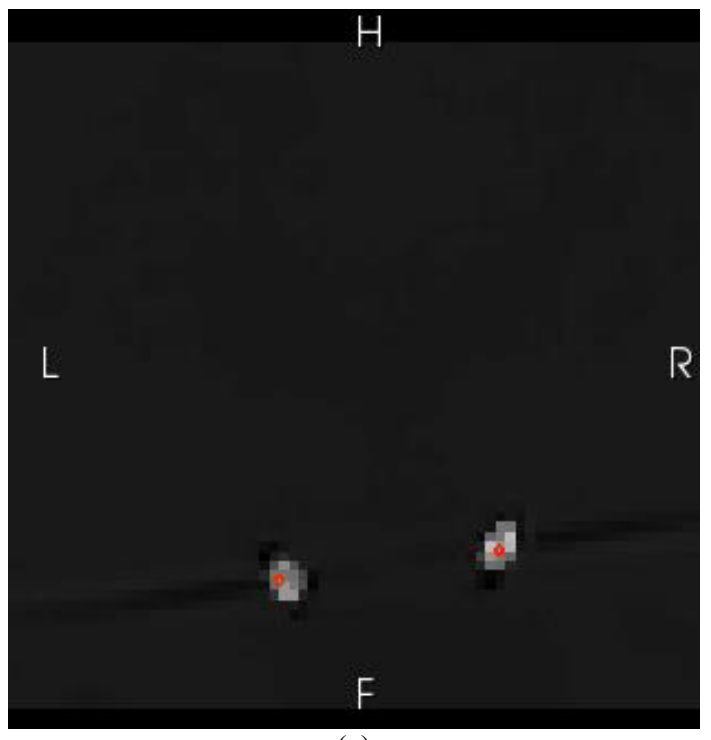

(c)

Figure 3: Contacts' centers in coronal (a), sagittal (b) planes, and the bounding box of sagittal plan (c) 


\subsection{Analysis of electrodes' deformation}

By comparing the average of mean curvature index for both sexes, we observed a greater MCI for female patients (using Multiple factor Analysis). In analyzing patients' data, we obtained a significant cross-correlation ( $\mathrm{r}=0.9$ ), between the mean curvature index of the electrode and the relative brain atrophy index for male patients ( $\mathrm{p}$-value $=0.002$ ) (see Figure 4). For female patients, we found a significant cross-correlation $(\mathrm{r}=0.5)$ between the mean curvature index of the electrode and the mean PD duration ( $\mathrm{p}$-value $=0.2$ ) (see Figure 5).

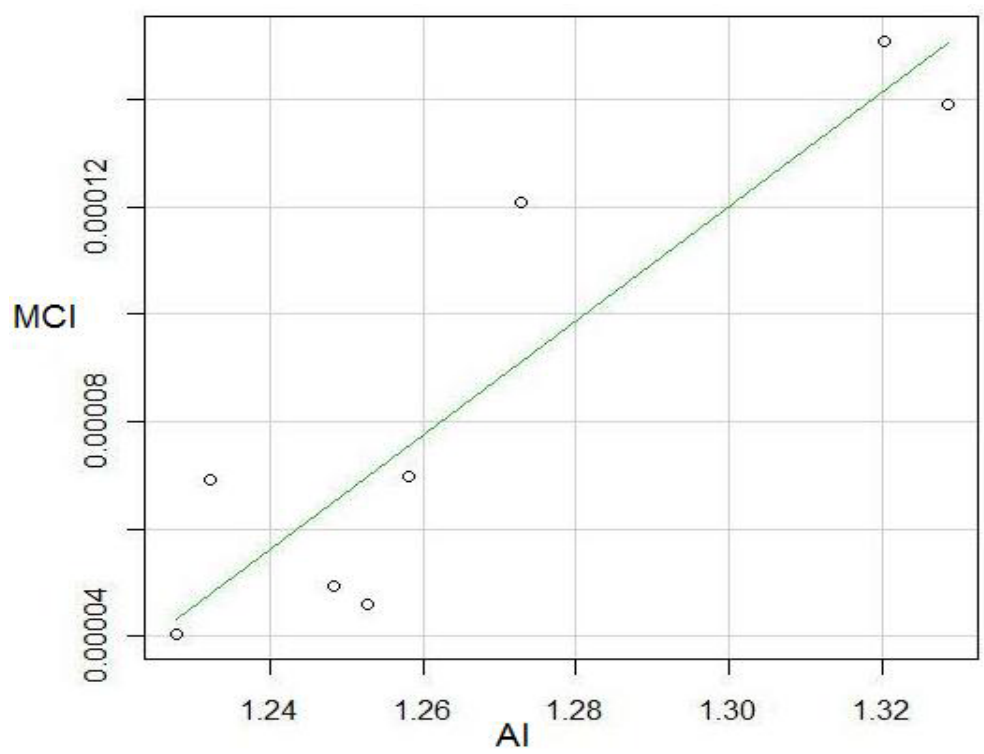

Figure 4 : Correlation between the mean curvature index of the electrode (MCI) and the relative brain atrophy index (AI) for male patients

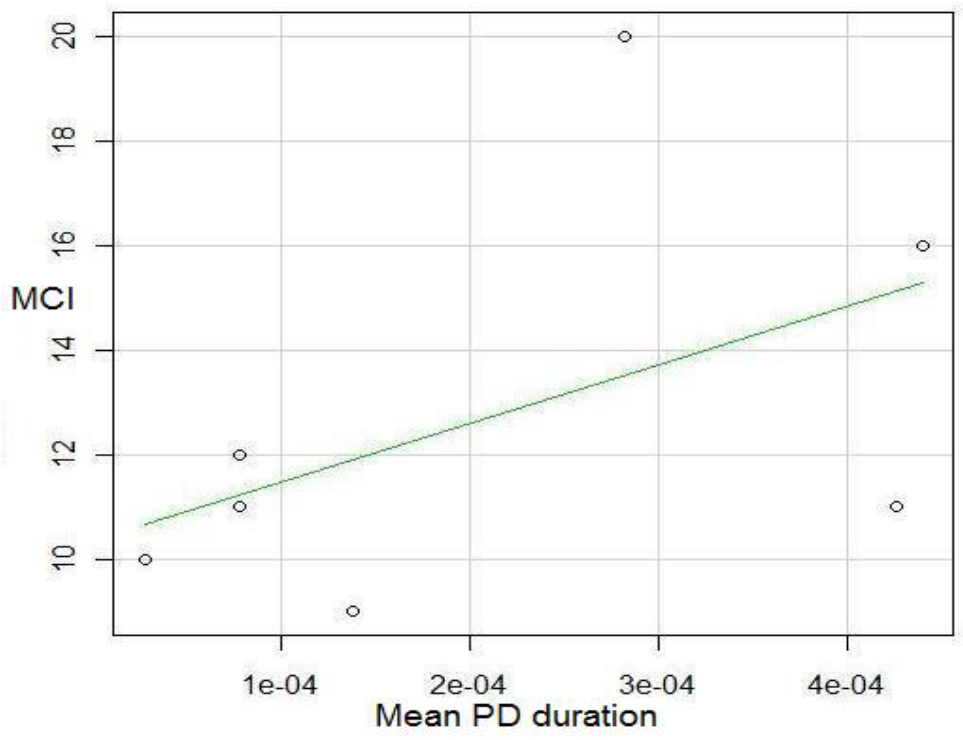

Figure 5: Correlation between the mean curvature index of the electrode (MCI) and the mean PD duration for female patients

We observed that the mean curvature index of the right electrodes (211.08e-06) was greater than the same index of the left electrodes (168.53e-06). By comparing relative brain atrophy index for both sexes, we found no significant difference (Kruskal-Wallis' test). 


\section{4 - DISCUSSION}

We demonstrated the accuracy of automatic contact localization algorithm based on electrode geometry. However, its robustness considerably depends on selected ROI. For three patients, the contact coordinates seemed to be aberrant due to an inaccurate local registration step. We are still working on development and improvement of our segmentation algorithm. Additionally, we found a greater correlation between the mean curvature index of the electrode and the relative brain atrophy index for male patients than for female patients and a positive correlation between the mean curvature index of the electrode and the mean PD duration for female patients. The greater mean curvature index was observed in female patients and on the right cerebral hemisphere in all patients. One assumption could be that the curvature is more dependent on cerebral density than on brain atrophy. Cerebral density could be lower in female than male and in the right hemisphere because of the non-dominant hemisphere in right-handed patients. However, further studies able to measure cerebral density are required for further analysis of this complex phenomenon.

\section{5 - CONCLUSION}

In this paper, we have reported on our current progress toward developing automatic contact localization algorithm and electrode curvature analysis. This yielded a correlation between the mean curvature index of the electrode, mean PD duration, and relative brain atrophy index according to patients' sexes. Correlation' results permitted to show that the electrodes' curvature increases with brain atrophy for male and mean PD duration for female patients. Such analysis would help anticipating electrodes' deformation and placement. Next studies will have to integrate more patients, and a digital atlas integrating patient's location of electrode contacts and clinical scores in order to evaluate DBS electrodes implantation taking into account motor or neuropsychological clinical scores ensuring the generation of rules for finding the optimum site for STN-DBS ${ }^{[19]}$.

\section{REFERENCES}

[1] Lang, AE. and Lozano, AM., "Parkinson's Disease", The New England Journal of Medicine, 339, 1044-1053 (1998).

[2] Benabid, AL., Krack, P., Benazzouz, A., Limousin, P., Koudsie, A. and Pollak, P., "Deep brain stimulation of the subthalamic nucleus for Parkinson's disease: methodologic aspects and clinical criteria", Neurology, 55, 40-44 (2000).

[3] Boisson, D., "Deep brain stimulation and Parkinson's disease", Neurol Neurosurg Psychiatry, 51, 491-500 (2008).

[4] Krack, P., Batir, A., Blercom, N., Chabardes, S., Fraix, V., Ardouin, C., Koudsie, A., Limousin, P., Benazzouz, A., Lebas, J., Benabid, A. and Pollak, P., "Five-year follow-up of bilateral stimulation of the subthalamic nucleus in advanced parkinson's disease", The New England Journal of Medicine, 349, 1925-1934 (2003).

[5] Saint-Cyr, J., Trepanier, L., Kumar, R., Lozano, A. and Lang, A., "Neuropsychological consequences of chronic bilateral stimulation of the subthalamic nucleus in parkinson's disease", Brain, 123, 2091-2108 (2000).

[6] Alegret, M., Junque, C., Valldeoriola, F., Vendrell, P., Pilleri, M., Rumia, J. and Tolosa, E., "Effects of bilateral subthalamic stimulation on cognitive function in Parkinson disease", Arch Neurol, 58, 1223-1227 (2001).

[7] Dujardin, K., Blairy, S., Defebvre, L., Krystkowiak, P., Hess, U., Blond, S. and Destee, A., "Subthalamic nucleus stimulation induces deficits in decoding emotional facial expressions in parkinson's disease", Neurol Neurosurg Psychiatry, 75, 202-208 (2004).

[8] Biseul, I., Sauleau, P., Haegelen, C., Trebon, P., Drapier, D., Raoul, S., Drapier, S., Lallement, F., Rivier, I., Lajat, Y. and Verin, M., "Fear recognition is impaired by subthalamic nucleus stimulation in parkinson's disease", Neuropsychologia, 43, 1054-1059 (2005).

[9] Theodosopoulos, P., Marks, W., Christine, C. and Starr, P., "Locations of movement related cells in the human subthalamic nucleus in parkinson's disease", Movement Disorders, 18, 791-798 (2003).

[10] Elias, WJ., Fu, KM. and Frysinger, RC., "Cortical and subcortical brain shift during stereotactic procedures", Neurosurg, 107, 983-988 (2007). 
[11] Miyagi, Y., Shima, F. and Sasaki, T., "Brain shift: an error factor during implantation of deep brain stimulation electrodes", Neurosurg, 107, 989-997 (2007).

[12] Munckhof, P., Contarino, M., Bour, L., Speelman, J., Bie, R. and Schuurman, R., "Postoperative curving and upward displacement of deep brain stimulation electrodes caused by brain shift", Neurosurgery, 67, 49-53 (2010).

[13] Coupe, P., Yger, P., Prima, S., Hellier, P., Kervrann, C. and Barillot, C., "An optimized blockwise nonlocal means denoising filter for 3-D magnetic resonance images", IEEE TMI, 24, 425-441 (2008).

[14] Powell, M., "The NEWUOA Software for Unconstrained Optimization without Derivatives", Workshop on Large Scale Nonlinear Optimization $\left(40^{\text {th }}\right)$, Italy, (2004).

[15] Wiest-Daesslé, N., Yger, P., Prima, S. and Barillot, C., "Evaluation of a new optimization algorithm for rigid registration of MRI data", SPIE: Medical Imaging, 6512, 601-610 (2007).

[16] Gray, A., Abbena, E. and Salamon, S., "Modern differential geometry of curves and surfaces with mathematica: The Gaussian and Mean Curvatures", Boca Raton, 2, 373-380 (1997).

[17] Ashbuurner, J. and Friston, K., "Unified segmentation", Neuroimage, 26, 839-851 (2005).

[18] Pollo, C., Villemure, JG., Vingerhoets, F., Ghika, J., Maeder, P. and Meuli, R., "Magnetic resonance artefact induced by the electrode activa 3389: an in vitro and in vivo study", Acta Neurochirurgica, 146, 161-164 (2004).

[19] Lalys, F., Haegelen, C., Abadie, A. and Jannin, P., "Correlating Clinical Scores with Anatomical Electrodes Locations for Assessing Deep Brain Stimulation", IPCAI, 6689, 113-121 (2011). 